\title{
Spatial distribution of disseminated histoplasmosis and AIDS co-infection in an endemic area of Northeastern Brazil
}

\author{
Francisco Gustavo Silveira Correia ${ }^{[1]}$, Carlos Henrique Alencar ${ }^{[1]}$, Lisandra Serra Damasceno ${ }^{[1]}$, \\ Isadora Cavalcanti Ramos ${ }^{[1]}$, Lícia Borges Pontes ${ }^{[1]}$ and Terezinha do Menino Jesus Silva Leitão ${ }^{[1]}$
}

[1]. Departamento de Saúde Coletiva, Faculdade de Medicina, Universidade Federal do Ceará, Fortaleza, Ceará, Brasil.

\begin{abstract}
Introduction: The spatial distribution of disseminated histoplasmosis (DH) and acquired immunodeficiency syndrome (AIDS) co-infection in adult residents of Fortaleza, Ceará, Brazil was evaluated. Methods: Socio-demographic data for the DH/AIDS cases were obtained from a reference hospital, and socio-environmental indicators were obtained from an official Brazilian institute. Kernel analysis and local indicators of spatial autocorrelation (LISA) cluster maps were used to estimate the case density within the city. Results: DH/AIDS cases were concentrated in the Northwestern and Southwestern peripheral areas of the city, related with low human development indices, but different from AIDS cases distribution. Conclusion: Risk factors other than AIDS infection must affect histoplasmosis development in this area.
\end{abstract}

Keywords: Disseminated histoplasmosis. AIDS. Spatial analysis.

Histoplasma capsulatum, considered the most common endemic mycosis in humans ${ }^{(1)}$, has emerged as an important opportunistic pathogen in individuals with impaired cellular immunity such as those undergoing prolonged corticosteroid and other immunosuppressive therapy, transplant recipients, those with advanced acquired immunodeficiency syndrome (AIDS), and more recently, those undergoing tumor necrosis factor antagonist treatment ${ }^{(2)}$. In Brazil, the State of Ceará had the highest number of deaths with histoplasmosis reported as the primary cause ${ }^{(3)(4)(5)(6)}$ than other endemic areas. Brilhante et al. ${ }^{(5)}$ conducted research with 208 patients with disseminated histoplasmosis and acquired immunodeficiency syndrome (DH/ AIDS) over a 5-year period in Ceará, resulting in an overall mortality rate of $42.3 \% \%^{(5)}$. A histoplasmin survey conducted with 161 HIV-positive patients with CD4 T-lymphocyte (CD4 cell) counts $>350$ cells $/ \mathrm{mm}^{3}$ who resided in Fortaleza, the capital of Ceará, found a $11.8 \%$ prevalence of reactivity suggesting important Histoplasma transmission in that area ${ }^{(7)}$. We aimed to investigate the role of the district-environmental and demographic factors in the spatial distribution of DH/AIDS cases in Fortaleza.

The study population consisted of patients diagnosed with AIDS according to the Brazilian Ministry of Health criteria ${ }^{(8)}$; with or without antiretroviral therapy (ARV); $>18$ years old; of either sex; residing in Fortaleza, the capital of Ceará

Corresponding author: Francisco Gustavo Silveira Correia.

e-mail: gustavcorreia@gmail.com

Received 19 September 2015

Accepted 14 March 2016
(Northeastern Brazil); and diagnosed with DH during hospital admission at São José Hospital (SJH), which is the reference hospital for infectious diseases in the State of Ceará, during the 1999-2007 period. DH was considered when H. capsulatum was identified by microscopy (histology or stained smears) and/or culture of the affected tissues or fluids outside the lungs and hilar lymph nodes.

Cases were identified using a databank of previous studies performed with patients with AIDS and DH at São José Hospital $^{(4)(9)}$. Socio-demographic data (age, sex, address, education level, birthplace, and income) were obtained from medical records; missing records were excluded from the analysis. Data relating to HIV infection comprised the timing of the AIDS diagnosis (if already diagnosed before hospital admission), CD4 cell count, previous admissions due to DH, and use of antiretroviral therapy or illicit drugs. Data regarding clinical outcomes (cure or death) were also collected. The following socio-environmental indicators were provided by the Brazilian Institute of Geography and Statistics [Instituto Brasileiro de Geografia e Estatística (IBGE) ${ }^{(10)}$ : resident population, which represents the number of persons residing in the neighborhoods in the macro-administrative regions of Fortaleza (regional), excluding the floating population; human development index (HDI), reflecting regional development that included aspects of the educational services (literacy rate, school enrollment), longevity of the population (life expectancy), and income; resident population by type of permanent private household (PPH), representing the number of persons residing in dwellings, subdivided into house, apartment, room, and improvised, by the resident population of a neighborhood; water supply index (WSI), representing the amount of private 
households supplied by water from the general network, by the total number of households in a neighborhood; regional WSI, which was obtained by averaging all of the WSIs from the neighborhoods; sewage index (SI), indicating the number of households covered by the general network of sewage collection divided by the total number of households in a certain neighborhood; and waste collection index (WCI), representing the number of households served by public cleaning services (garbage collected), by the total number of households in a neighborhood. These data were compiled with georeferenced maps to evaluate the spatial distribution of $\mathrm{DH}$. According to the IBGE, Fortaleza is the fifth most populated Brazilian city, with an estimated 2,551,805 inhabitants and a population density of $8,102,77$ inhabitants $/ \mathrm{km}^{2}$, corresponding to approximately $30 \%$ of the state population ${ }^{(10)}$.

For the spatial analysis, we adopted the districts as the unit of analysis. Fortaleza is divided into 116 districts, grouped in six macro-administrative regions that are denominated Secretarias Executivas Regionais (SER). Descriptive spatial analysis was modeled by applying the Kernel estimator and empirical Bayes smoothing techniques. The spatial analysis methods used to evaluate the spatial dependency of the cases were the Global Moran's I index, which auto-correlated variables spatially to indicate a similarity, or lack of similarity, between the neighbors, and the local Moran Index (LISA), which allowed the identification of significantly high values and positive clusters of both high and low values ${ }^{(11)}$. This analysis was performed using the program Terraview (version 4.0.0) and the LISA cluster map, which shows only neighborhoods with statistical significance $(p<0.05)$ classified into four possibilities: highhigh and low-low, representing spatial units with a high/low frequency of cases and neighboring units also with a high or low frequency, and high-low and low-high, representing units with high and low frequencies surrounded by units with low and high frequencies ${ }^{(12)}$. The research protocol 041/2011 was reviewed and approved by the Ethical Committee of the São José Hospital. No variables allowed identification of individuals.

We identified 264 patients with progressive (or acute) DH/AIDS co-infection for the period 1999-2007. Of these, 152 patients were residents of Fortaleza, which 43.4\% were born in the city; after excluding 17 patients with missing records, 135 patients $(82.2 \%$ men $)$ were included in the analysis. The patients had a mean age of 40.3 years, 4-7 years of schooling (34.8\%), and a monthly income $(51 \%)$ of $<3$ minimum wages of approximately 1140.00 USD in 2007 (51\%). AIDS was diagnosed because of illness with DH for 62 patients (45.9\%); of these patients, $59(43.7 \%)$ were undergoing highly active anti-retroviral therapy (HAART). The average CD4 count at discharge in patients with DH was 103.4 cells $/ \mathrm{mm}^{3}$ ([standard deviation (SD)], 161.2); 126 (93.3\%) patients had not been admitted previously for $\mathrm{DH}$. Illicit drugs were used by 62 (45.9\%) patients, and $30 \%$ of the patients died during the study period. The average CD4 count of the patients who died was $117.7 \mathrm{cell} / \mathrm{mm}^{3}$ (SD, 124.2).
The analysis included 75 (64.7\%) districts of Fortaleza and all 6 SERs. The cumulative proportion identified districts with high numbers of DH cases, with the largest sample located in the Southwestern $(21.8 \%$ of cases) and Northeastern outskirts of the city (Figure 1). The socioenvironmental indicators showed low sewage coverage in SERs IV (22\%) and V (28.9\%). Only SER II had a medium-level HDI (0.693); all other SERs had low HDIs $(<0.499)$. The WSI and WCI were considered high $(>90 \%)$ in all regions (Table 1).

The spatial distribution of DH/AIDS cases using the Kernel estimator map showed districts with a high frequency corresponding to areas that composed SERs I, III, and IV. The point cloud extended throughout town, showing hotspots in the Northwest of the city (Figure 1). LISA cluster maps of the DH/AIDS case distribution showed geographical confluences in the reported results, with high rates concentrated especially along the peripheral western edge of the town. Thematic maps, which deal with point cases and environmental factors, showed that the northwestern areas had a high number of cases and medium-level SI and HDI. A high density of houses, rather than apartments (PPH >67\%), was observed with these analyses (Figure 2).

Since the beginning of the AIDS epidemic, DH has been detected with increasing frequency in patients attending the reference hospital for HIV in Ceará( ${ }^{(6)}$. This probably reflects the increasing incidence of AIDS cases in Fortaleza ${ }^{(12)}$, instead of increased fungal dissemination in that area.

The socio-demographic profile of the patients is similar to that in the AIDS epidemiological bulletin of the local health department, which showed that the cumulative number of AIDS cases was present primarily in men (73.3\%), with 4-7 years of education (23.7\%) during 1983-2009 period $^{(12)}$. The proportion of patients who were diagnosed with HIV/AIDS due to illness with DH (>54\%) is also similar to that reported previously ${ }^{(4)}$.

The Northwestern (SER I) and Southwestern (SER V) peripheral areas of the city were the regions with higher frequencies of DH/AIDS cases. The spatial distribution of AIDS cases in Fortaleza between 2000 and 2008 showed that AIDS patients were primarily in the mideastern part of the city (SER III) ${ }^{(12)}$, which does not reflect the distribution of DH/AIDS cases in the present study, indicating that other risk factors beyond AIDS affect the spatial distribution of $\mathrm{DH}$ in the city.

Secretaria Executiva Regional V, the most populous area (452,875 inhabitants), included the districts with low sewage coverage (28.9\%) and lower HDI, while SER I had higher SI (57.9\%) and WCI (96.9\%). In SER I, there are probable specific environmental factors for the spread of 


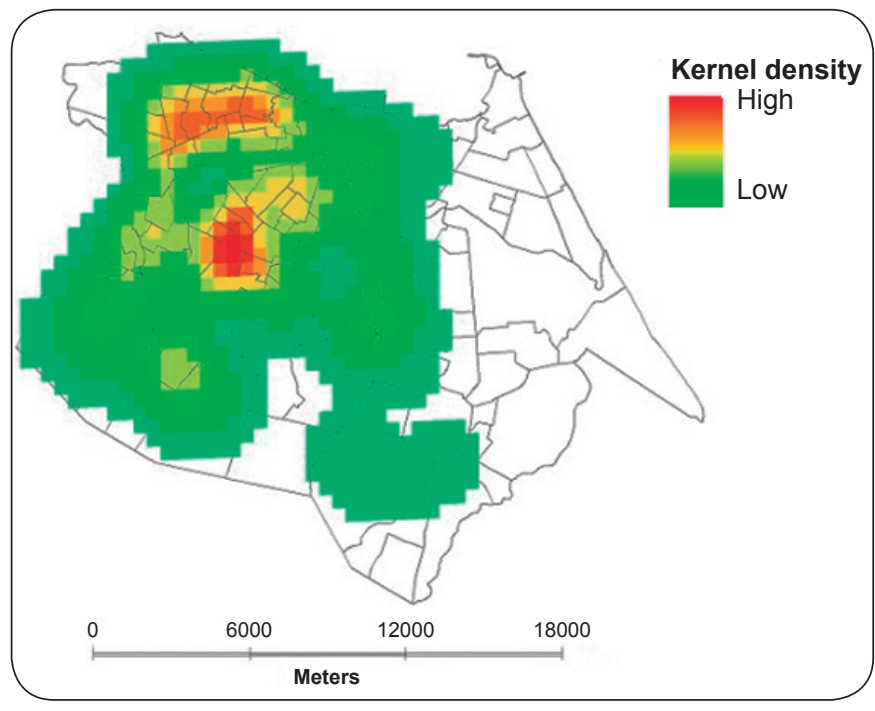

FIGURE 1 - Kernel estimator map of the cumulative frequency of disseminated histoplasmosis and acquired immunodeficiency syndrome co-infection in Fortaleza, Ceará, Brazil, 1999-2007.

histoplasmosis, that are not involved with sanitation which should be investigated, such as the presence of bat or bird droppings. SER I had some characteristics that can favor the presence of Histoplasma; it is surrounded by the Ceará River and has an area of environmental preservation for ecotourism and fishing. It has also an area of mangroves with birds, which should be investigated for the presence of the fungus. Furthermore, the low sanitation coverage in SER I can encourage the presence of birds and bats, producing conditions for human infection.
Areas with traditional houses can maintain some common dissemination methods for fungus such as soil manipulation and breeding of birds and tree crops for landscaping or eating. The presence of mango trees (Mangifera indica) and activity involving soil were significantly associated with histoplasmin reactivity in a study performed with $161 \mathrm{HIV}$ patients residing in Fortaleza ${ }^{(7)}$. Mango trees can also be related with the presence of bats; these animals are attracted by its fruit and can live for years in the nearest places to roost such as the roofs of homes, promoting areas of accumulated organic matter and manure, an excellent place for $H$. capsultaum proliferation ${ }^{(2)(7)(13)}$.

In another study by Cordeiro et al. ${ }^{(14)}, H$. capsulatum was not isolated from 83 bats that were captured from the countryside looking for fungus, but Coccidioides posadasii was recovered from Carollia perspicillata in bat lungs; no information concerning bats captured from Fortaleza has been published ${ }^{(14)}$.

Analyses using the LISA showed significant spatial clusters (high values surrounded by high values) in SERs $\mathrm{I}$ and $\mathrm{V}$, demonstrating areas of a high number of cases exhibiting low and medium sanitation coverage and medium HDIs, reinforcing that other factors are involved in co-infection.

The results of the 2000 Census were highly reliable owing to the use of modern technologies such as digital mapping of the municipalities, scanning and optical reading of the questionnaires, and managerial and operational controls over the internet, among other technological innovations ${ }^{(15)}$.

TABLE 1 - Distribution of cases of disseminated histoplasmosis/AIDS co-infection by year of occurrence and socio-environmental indicators by Secretarias Executivas Regionais code.

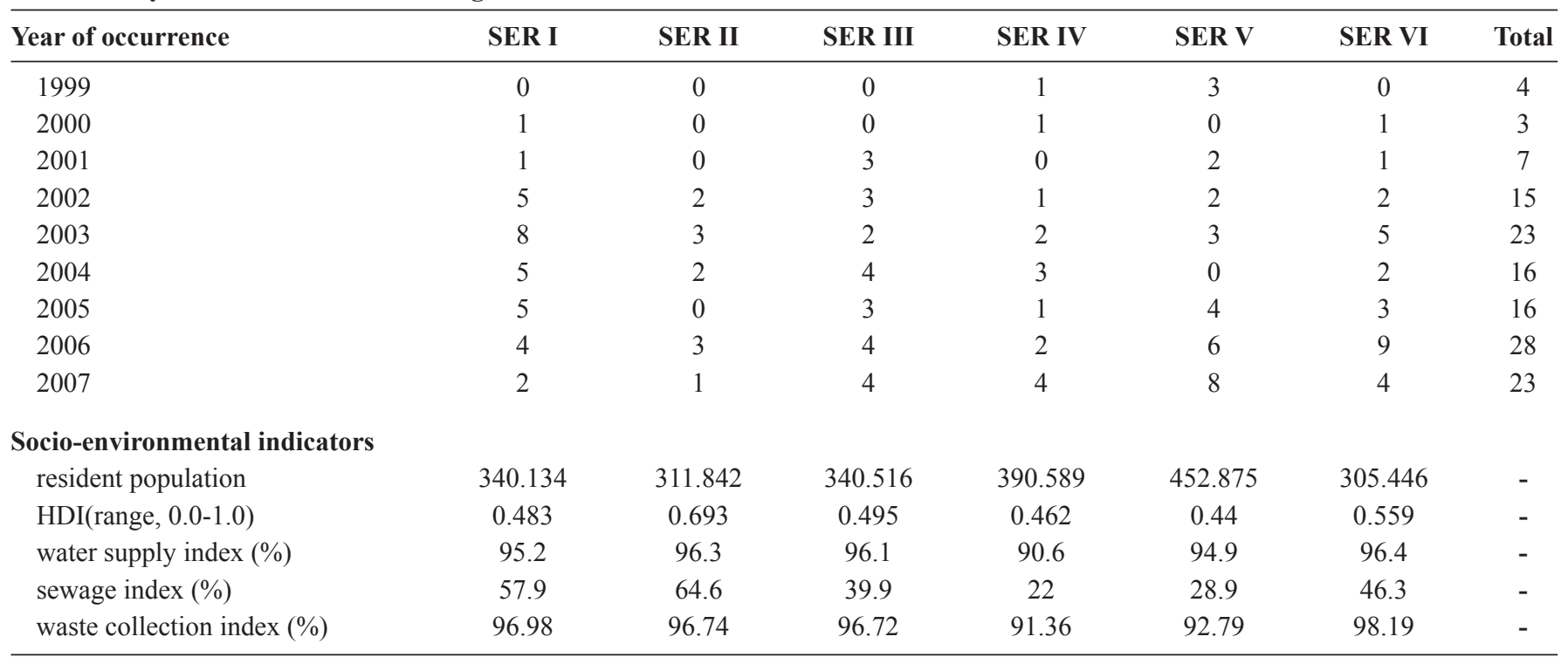

AIDS: acquired immunodeficiency syndrome; SER: Secretaria Executiva Regional; HDI: human development index. 

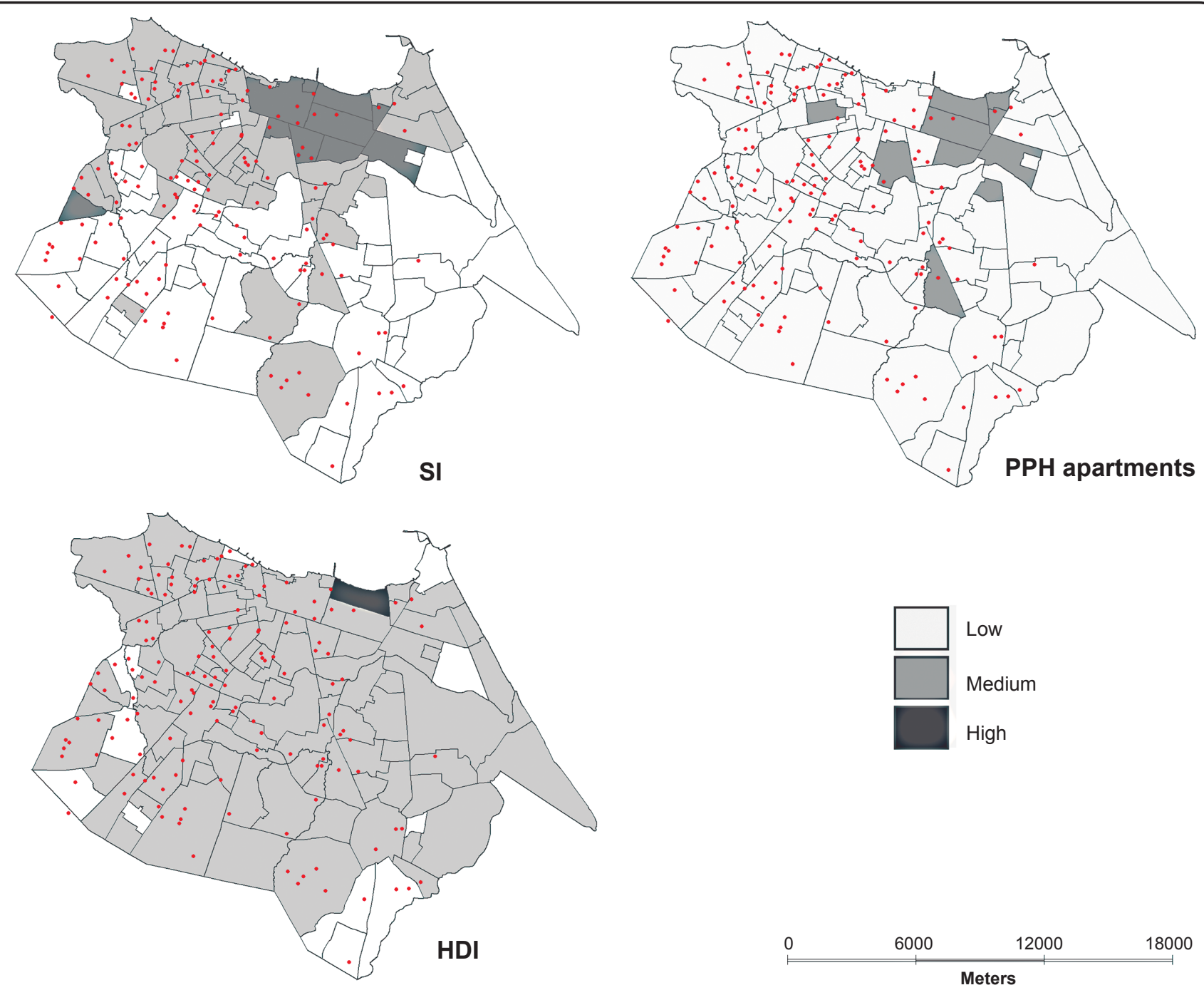

FIGURE 2 - Cases of disseminated histoplasmosis/AIDS co-infection and the sewage index (SI), human development index (HDI), and permanent private households (PPH) in the form of apartments, by district in Fortaleza, Ceará, Brazil, 1999-2007.

However, this study also had some limitations. The information was obtained from secondary data, which could limit the reliability of the results. Because the 135 patients are not likely representative of the estimated frequency of DH in Fortaleza, any extrapolation to the general population of patients with DH and AIDS should be conducted with caution. All patients diagnosed with $\mathrm{DH}$ were residents of Fortaleza; however, we cannot affirm that these individuals were infected in the city. Furthermore, a considerable number of cases were born in the countryside.

The need to study the relation between human beings and the environment resulted in several studies of medical geography, aimed at analyzing disease spatial distributions ${ }^{(15)}$. However, this is the first description of the spatial distribution of DH/AIDS co-infection and the relationship with environmental factors, allowing a different viewpoint of the morbidity related with this severe disease in an endemic area. The distribution of cases overlaying areas with low sanitation coverage and HDI indicated that other risk factors beyond AIDS must affect the development of histoplasmosis in these studied areas.

\section{ACKNOWLEDGMENTS}

We are very grateful to Gauss Statistical Solutions for the assistance with the spatial analysis as well as the physicians, nurses, and technicians from the Infectious Diseases São José Hospital for the assistance during data collection. 


\section{FINANCIAL SUPPORT}

We are thankful to the Fundação Cearense de Apoio ao Desenvolvimento Cientifico e Tecnológico (FUNCAP) for the financial support.

\section{CONFLICT OF INTEREST}

The authors declare that there is no conflict of interest.

\section{REFERENCES}

1. Wheat LJ. Approach to the Diagnosis of the Endemic Mycoses. Clin Chest Med 2009; 30:379-389.

2. Kauffman CA. Histoplasmosis. Clin Chest Med 2009; 30:217-225.

3. Prado M, Silva MB, Laurenti R, Travassos LR, Taborda CP. Mortality due to systemic mycosis as a primary cause of death or in association with AIDS in Brazil: a review from 1996 to 2006. Mem Inst Oswaldo Cruz 2009; 104:513-521.

4. Pontes LB, Leitão TMJS, Lima GG, Gerhard ES, Fernandes TA. Clinical and evolutionary characteristics of 134 patients with disseminated histoplasmosis associated with AIDS in the state of Ceará. Rev Soc Bras Med Trop 2010; 43:27-31.

5. Brilhante RSN, Fechine MAB, Mesquita JRL, Cordeiro RA, Rocha MFG, Monteiro AJ, et al. Histoplasmosis in HIV-positive in Ceará, Brazil: clinical-laboratory aspects and in vitro antifungal susceptibility of Histoplasma capsulatum isolates. Trans Royal Soc Trop Med Hyg 2012; 106: 484-488.
6. Damasceno LS, Ramos AN, Alencar CHM, Lima DT, Sidrim JJC, Gonçalves MVF, et al. Disseminated histoplasmosis and aids: relapse and late mortality inendemic area in north-eastern Brazil. Mycoses 2013; 56: 520-526.

7. Bezerra FS, Zancopé-Oliveira RM, Brilhante RSN, Wanke B, Mota RMS, Ramos APG, et al. Histoplasmin survey in HIVpositive patients: results from an endemic area in northeastern Brazil. Rev Inst Med Trop São Paulo 2013; 55:261-265.

8. Ministério da Saúde. Secretaria de Vigilância em Saúde. Programa Nacional de DST e Aids. Critérios de definição de casos de AIDS em adultos e crianças. Brasília: Ministério da Saúde, 2003.

9. Ramos IC. Clinical laboratory findings helping the diagnosis of disseminated histoplasmosis in patients with febrile AIDS in a reference hospital of Ceará. 2008. 134p. (Master's dissertation). Departamento de Saúde Coletiva. Federal University of Ceará; 2008; Ceará.

10. Instituto Brasileiro de Geografia e Estatística (IBGE). Censuses. Acessed on 12 December 2012 at $<$ http://www.ibge.gov.br/home/ estatistica/populacao/default_censo_2013.shtm>.

11. Druck S, Carvalho MS, Câmara G, Monteiro AVM. Análise Espacial de Dados Geográficos. Brasília: Embrapa; 2004.

12. Ceará. Secretaria Municipal de Saúde de Fortaleza. Coordenação Municipal de DST, Aids e Hepatites Virais. Boletim Epidemiológico DST/AIDS. 2009; 13, nº3.

13. Guimarães AJ, Nosanchuck JD, Zancopé-Oliveira RM. Diagnosis of histoplasmosis. Braz J Microbiol 2006; 37:1-13.

14. Cordeiro RA, Silva KRC, Brilhante RSN, Moura FBP, Duarte $\mathrm{NFH}$, Marques FJF, et al. Coccidioides posadasii infection in Bats, Brazil. Emerg Infect Dis 2012; 18:668-670.

15. Hino P, Villa TCS, Cunha TN, Santos CB. Distribuição espacial de doenças endêmicas no município de Ribeirão Preto (SP). Ciência \& Saúde Coletiva 2011; 16 (supl 1):1289-1294. 